\title{
Top-Down Design Methodology \& Technology for Microsystems
}

Jordi Carrabina ${ }^{\prime}$, Joaquin Saiz', David Marin ${ }^{1}$, Xavier Marin ${ }^{\prime}$, Angel Merlos', Joan Bausells ${ }^{2}$

${ }^{1}$ U. Microeletrònica. Dpt. Informàtica. Universitat Autònoma de Barcelona.

${ }^{2}$ Centre Nacional de Microelectrònica. CNM-IMB (CSIC).

Campus UAB. 08193 Bellaterra. Spain.

\begin{abstract}
The evolution of microeletronic design techniques implies an important methodological effort to increase the degree of abstraction far from technology and devices. This process is not uniform and depends on the specific electronic domains. As for sensors and actuators, there has been quite a few approaches toward this global system design methodologies, and most of them in the last years. The main points for this relative delay are: non-standard technological processes, specially in case of embedded microsystems, different types of analysis required, that use different environments for each magnitude (mechanics, flow, magnetic,..), connection between circuit and structural 3D simulators, whose representations need to be abstracted by introducing the concept of sensor cell libraries, to estimate performance in the global system (large areas, signal sensitivities, mechanical restrictions,...). In the paper, we show the choices we select for these decisions in the case of a new microsystem fabrication process for embedded microsystems, developed together with the tool and design methodology, based on the 1.0 $\mu \mathrm{m}$ CMOS from ATMEL-ES2. Some demonstrators are presented, together with measurement results.
\end{abstract}

\section{Keywords}

Microsystems Design, CAD tools for MEMs, Microsystems Technology. 


\section{INTRODUCTION}

Sensors and actuators have been driving the evolution of electronics, since they are present in most of the industrial products. This large market suffered from an impressive evolution in the last years due to the requirements of new applications like safety in cars, water saving and water pollution, spatial applications, home systems, etc. Often, those sensors are not interfaced with simple electronics, but with complex systems through specific buses. Also, area and weight need to be reduced for many applications.

All these requirements, directed the evolution of microsystems technology during the last years, Baltes (1193), with different alternatives: monolithic implementations, where sensor and electronics share the same silicon substrate, multi-chip modules MCM, which contain several specialized chips for sensing and processing, and "classical" PCB-based system.

From the point of view of a system designer, the ideal tool has to offer the possibility to perform a given analysis of the system modeled at very high level, while keeping the main constrains of the implementation (price, speed and power consumption). In order to minimize cost functions for complex systems the designer has to work even at low levels of abstraction, down to full-custom level.

For the case of microsystems design, Huijsing (1994), different types of analyses have to be carried out to ensure stability (i.e. AC analysis) and performance (i.e. DC analysis) of sensors. Most of that work is being done by structural 3D simulators.

Interactions between different subsystems (sensor, analog and digital) have to be taken into account in the design process to produce the whole system. The complexity, at the system level, of digital parts is driving the global verification process, usually through transient simulation. This analysis has to be enough accurate to cope with small sensor magnitudes and dependencies, what usually lets to mixed-mode multi-level simulation, thus higher levels of description for sensors have to be available to system designers.

In this paper, we present our developments that concurrently try to develop a new approach for monolithic microsystems integration from both technology and design sides. In chapter 2, different sensor types are described, that correspond to different technological developments (related to different process steps). In chapter 3 , the design methodology is presented, following the steps of a "standard" bottom-up design methodology: full-custom, sensor cell libraries and semi-custom. Chapter 4 shows some circuits and the measured results for devices and microsystems, including a demonstrator designed for the prototype washing machine from Fagor Sensores (COPRECI). 


\section{SENSOR TYPES}

The standard process used is $1.0 \mu \mathrm{m}$ CMOS N-well from ATMEL-ES2, with one polysilicon and two metal layers. A post-processing technology has been designed at CNM, to allow fabrication of devices based in micromachined membranes, of a thickness up to $10 \mu \mathrm{m}$. Membranes are obtained by anisotropic etching from the back side of the wafer, using an additional mask, and a double-side aligned photolithographical step. With a further step, it is possible to etch devices from the front side, and then spring-mass structures can be obtained.

\section{Pressure sensors}

Piezoresistive pressure sensors have been fabricated by using this post-processing technology. The piezoresistors are obtained from the standard PMOS implant used for transistor source and drain, for thicker membranes $(2,5$ to $10 \mu \mathrm{m})$ and from doped polysilicon for thinner ones (up to 2,5 $\mu \mathrm{m}$ ), e.g. Marco (1993). Pressure sensors with $600 \times 600 \mu \mathrm{m}^{2}$ membrane, $5 \mu \mathrm{m}$ thickness are currently working for a 0.3 bar range, with $20 \mathrm{mV} / \mathrm{V} / \mathrm{bar}$ sensitivity and non-linearity lower than $0.4 \%$.

\section{Accelerometers}

Pressure sensors are based on a single membrane, while accelerometers use big mass of silicon, connected to the main silicon part that contains the circuitry, with small bridges of the same thickness that the membrane of pressure sensors where piezoresistors are placed. These structures have been successfully fabricated, and preliminary measurements of such device have shown satisfactory operation, though the design should be improved in order to get a larger range of operation and higher sensitivity. Competition with capacitive-type accelerometers seems to be difficult, though the process and integration with simple CMOS technologies is easier.

\section{ISFET Device}

The structure uses floating polysilicon with two metal layers on top (including stacked via and contact). Therefore, ISFET fabrication is fully compatible with the CMOS process (with some allowed design rule violations) and, consequently, its integration together with the technology is straightforward, e.g. Merlos (1995).

Implemented devices show mean sensitivities of $41 \mathrm{mV} / \mathrm{pH}$. Packaging is done on special PCB strips. An isolating material is used for the protection of both active devices and bonding area placed around the sensor device $\left(1 \mathrm{~mm}^{2}\right)$.

Figure 1 shows different aspects of those devices: membrane cavity from pressure sensors, floating mass of accelerometer, and profile of different layer for ISFET devices. Standard size of devices is shown on each SEM photograph. 

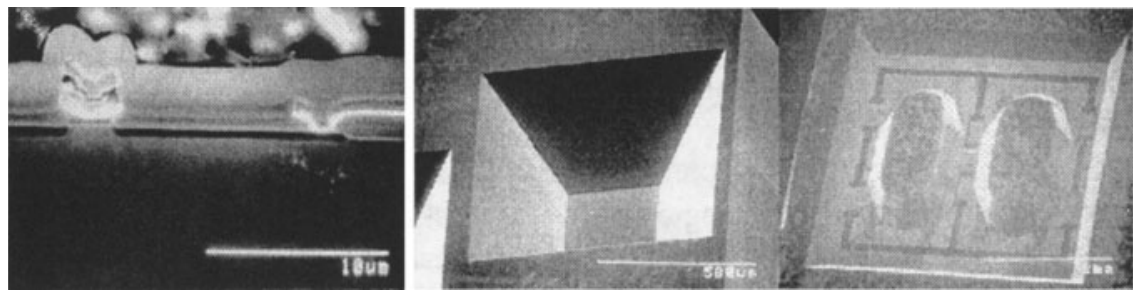

Figure 1. Devices obtained from standard $1 \mu \mathrm{m}$ CMOS process plus additional processes for pressure sensors (left), accelerometers (center) and ISFET (right).

\section{DESIGN KIT \& METHODOLOGY}

ASIC design methodology requires that customers be responsible for, at least, giving an structural description of their products. In this case, they only require front-end tools to define their designs. Back-end processes are either done by customers or by the foundry. Finally, layout generation is usually done by the foundry. Nevertheless, sometimes full-custom design is still required. This can often happen in the case of sensor design, because of the specificity of the sensor devices and their range of operation.

Design methodology offers the way to go from specifications to the layout. The border between design and technology is sensor cell library for the "front-end only" design case, and technology file for the complete design flow one. A similar approach can be found in Karam (1996), main differences relate the set of sensors (only front side etching is allowed) and the framework used (Mentor Graphics).

\subsection{Full-custom design environment: technology file}

Full-custom design requires a set of design rules for the different masks plus the physical parameters for the given simulator or device model. Basic tools from a framework can easily cope with this enhancements, by adapting the technology file for all geometrical definitions and relations, and simulation environment that allow the integration or link with additional simulators.

Sensor layouts are drawn using different geometries depending on the type of sensor. For instance, due to anisotropic properties of silicon, drawings on the membrane layer are considered different for pressure sensors and accelerometers, that include additional relations between membrane and passivation masks.

Therefore, new technology file covers: (1) the definition of design rules for the membrane mask, (2) the redefinition of rules for the rest of layers inside the sensor region, (3) and any specific restriction concerning the type of sensor.

Classical methods for the definition of design rules use creation and alignment tolerances depending on the machinery and degree of confidence. For 
microsystems, most of the values related to new masks are quite large if compared to the critical dimensions for standard layers. The geometry imposed by design rules related to back side etching is also quite large. This is because most of the instrumentation is not optimized for those new processes: double side alignment, electrochemical etch stop, whole wafer orientation according to crystallographic axes of the silicon, under-etching on back-side mask material.

All those aspects are taken into account, for each sensor type, in our current technology file (standard term for Cadence DFWII) through data concerning layer information, device definition, rules for DRC, extraction \& LVS rules, and abstract generation.

In the case of the device extractor, and layout versus schematic tool (LVS), the sensor cell has to be defined in advance, in order to extract the geometric parameters according to the device model. For instance, for the pressure sensor, we consider as a library elements both a single piezoresistive element and a symmetric Wheatstone bridge of piezoresistors (Figure 2).

For the floor-plan of microsystems composed of sensor devices and circuitry, the role of sensor is also important, since they cannot be placed anywhere. There are different types of physical restrictions: distance between sensor and rest of the circuit, pads or boundary. First two are related with changes in the behavior of electrical and bonding properties, while the last one is related to mechanical properties for packaging sensors.

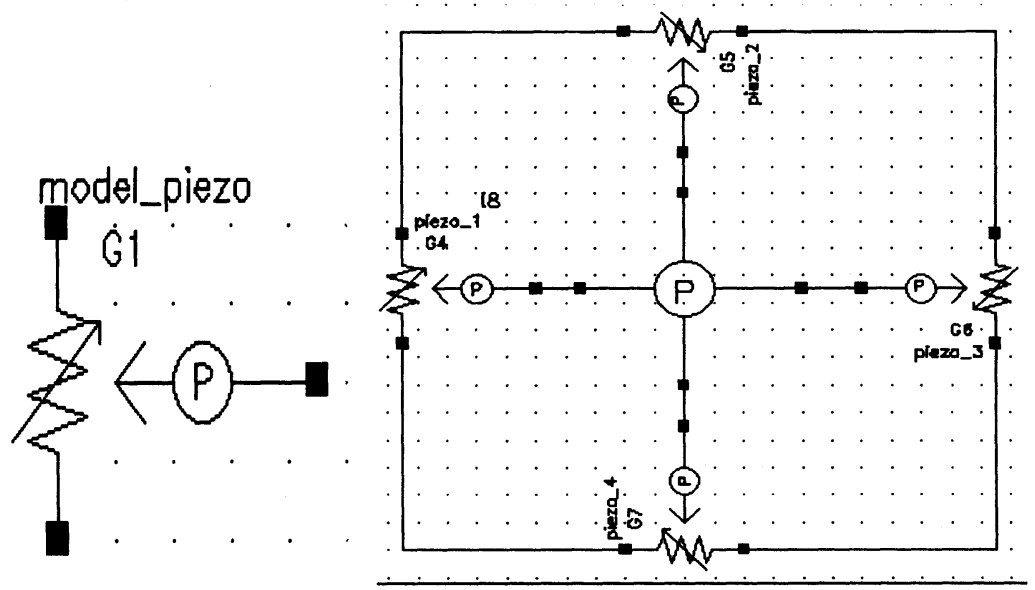

Figure 2. Symbols for piezoresistive device (left) and Wheatstone bridge (right).

Furthermore, there are electrical constrains, similar to the classical ones concerning mixed A/D ICs such as couplings, parasitic resistances,... that tend to minimize the 
distance and crossing of sensitive lines, and make the device as much symmetric as possible. A layout example for pressure sensor in shown in Figure 3 (a).

\subsection{Semi-custom environment}

ASIC design methodologies are based on handling elements described at higher degree of abstraction, and related CAD tools evolved to integrate different description levels (behavioral, structural, physical) into the same environment. In our case, microsystems contain a reduced number of devices, included in a basic sensor cell library of parameterizable devices, and with the appropriate CAD tools.

Our semi-custom environment also includes analog cells designed to meet specific electrical constraints of sensors (low signal level, impedance coupling,...).

\section{Device model generation}

For the design and improvement of any sensor, device engineers usually work with 3D structural simulators (i.e. Ansys) or derived environments, e.g. Korvin (1996). For instance, they derive some mechanical properties (stress) of mobile structures in silicon, and from that stress, taking into account piezoelectric properties of materials, it is possible to get their equivalent electric signals for each device. At this level, many different types of analyses can be performed (static, dynamic, harmonic, thermal,...).

Different authors propose mixed-mode simulation techniques to integrate this kind of simulators with electrical or HDL ones. The drawback of this global simulation is that computation through finite element modeling require a large amount of memory and CPU time, apart from the economic cost of those simulators.

Therefore, in order to use standard frameworks for system level design, a higher degree of abstraction should be used, after the initial modeling of sensor cells for the required analysis. These models can be derived either from some analytic theory, which can be easily implemented either using mathematical solvers (i.e. Mathematica), or from behavioral models. Both approaches require basic process parameters, and device parameters. In the end, computational cost and price for the customer are made smaller.

The critical point is the agreement between simulation and measurement. However, once process parameters are characterized, differences are not so important, and most of sources of non-ideal behavior can be easily introduced in the model (e.g. misalignment between piezoresistors and border of membrane).

This approach has other advantages related CAD tools, like: (1) Portability, because $C$ language is used for the sensor models; (2) wide range of application since starting from high level specifications (sensitivity, range,...) representations at different abstraction levels are obtained, (3) Basic equations for sensors are relatively simple (polynomials), and therefore convergence problems are reduced; 
558 Part Twelve Testing in Complex Mixed Analog and Digital Systems
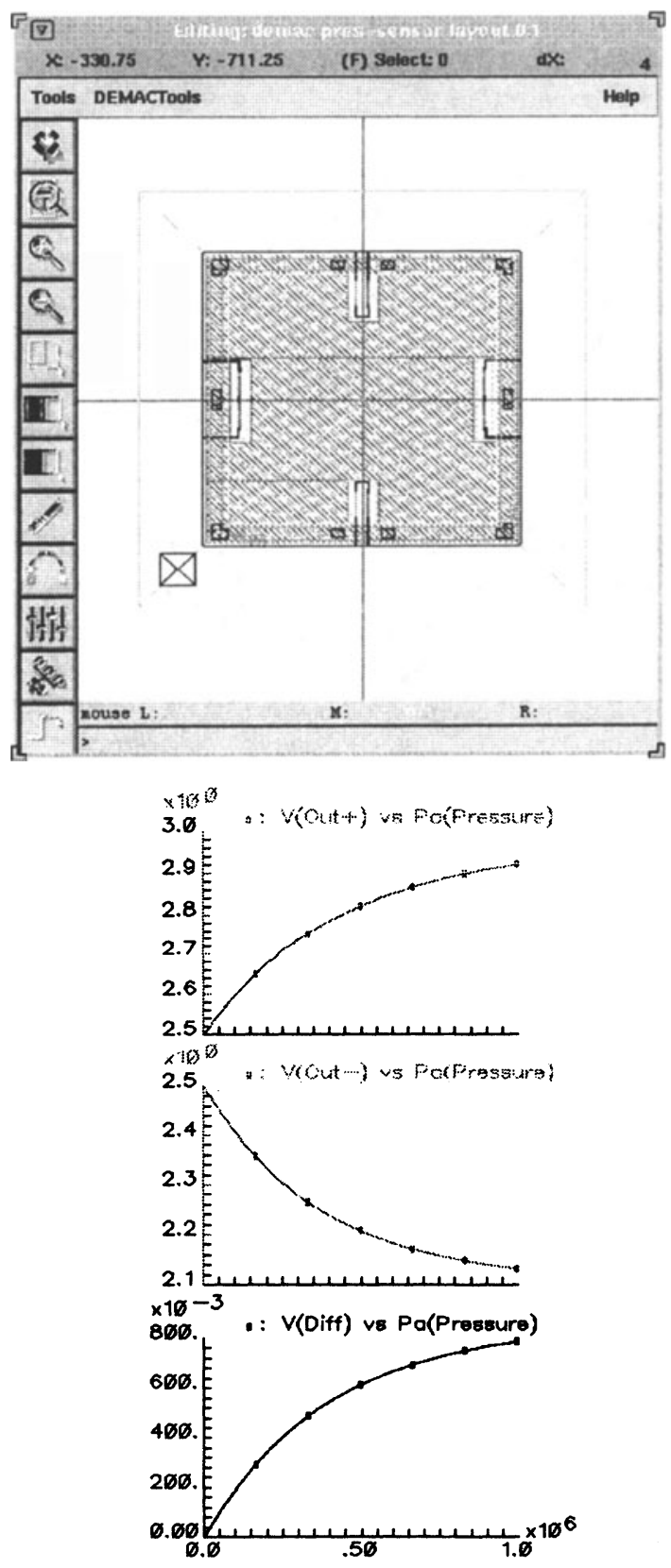

Figure 3. Pressure sensor: (a) Layout with four piezoresistors inside n-well and membrane, and (b) voltage of two outputs (top and center) and bridge (bottom). 
(4) Different models can be used for the same sensors according to the different working conditions (defined for each application).

For instance, pressure sensors can be modeled at 4 different levels for static analysis, Hebrard (1995):

- Level 1. Linear plate theory, with first order piezoresistive coefficients and simple temperature dependence.

- Level 2. Precise model for temperature, based in an electrothermal coupling.

- Level 3. Large defections consideration. Sensor output is represented by a look-up table Vout $=f(P, T)$.

- Level 4. Similar to the previous one, taking into account second order piezoresistive coefficients. An example is shown in Figure 3 (b). 
The choice of the model depends on the pressure range, and on the importance of the precision for the application (in order to include second order terms). These models use different parameters related to: (1) technology dependencies, used for process and device optimization, and fitting between measures and simulation results, (2) sensor type, according to specific predefined structures and active materials, and (3) design parameters, concerning the geometric values of devices, that can be extracted from layout views. Figure 4 shows the from that appears in the Cadence framework for model compilation.

Different models can be generated in a spice-like format and they are integrated in Design Framework II. Following a similar scheme Verilog models can be produced (currently, a standard for ATMEL-ES2 cell libraries). Difference between both types of models relates on the mathematical or tabular functions available to describe the model, what is important for sensor design. Nevertheless, it is even more important to work at the right level of precision, required for the system design. This level will be fixed be the application, and in most of the cases will require mixed-mode simulation composed of electrical models for sensor and analog parts, and HDL models for digital ones. 


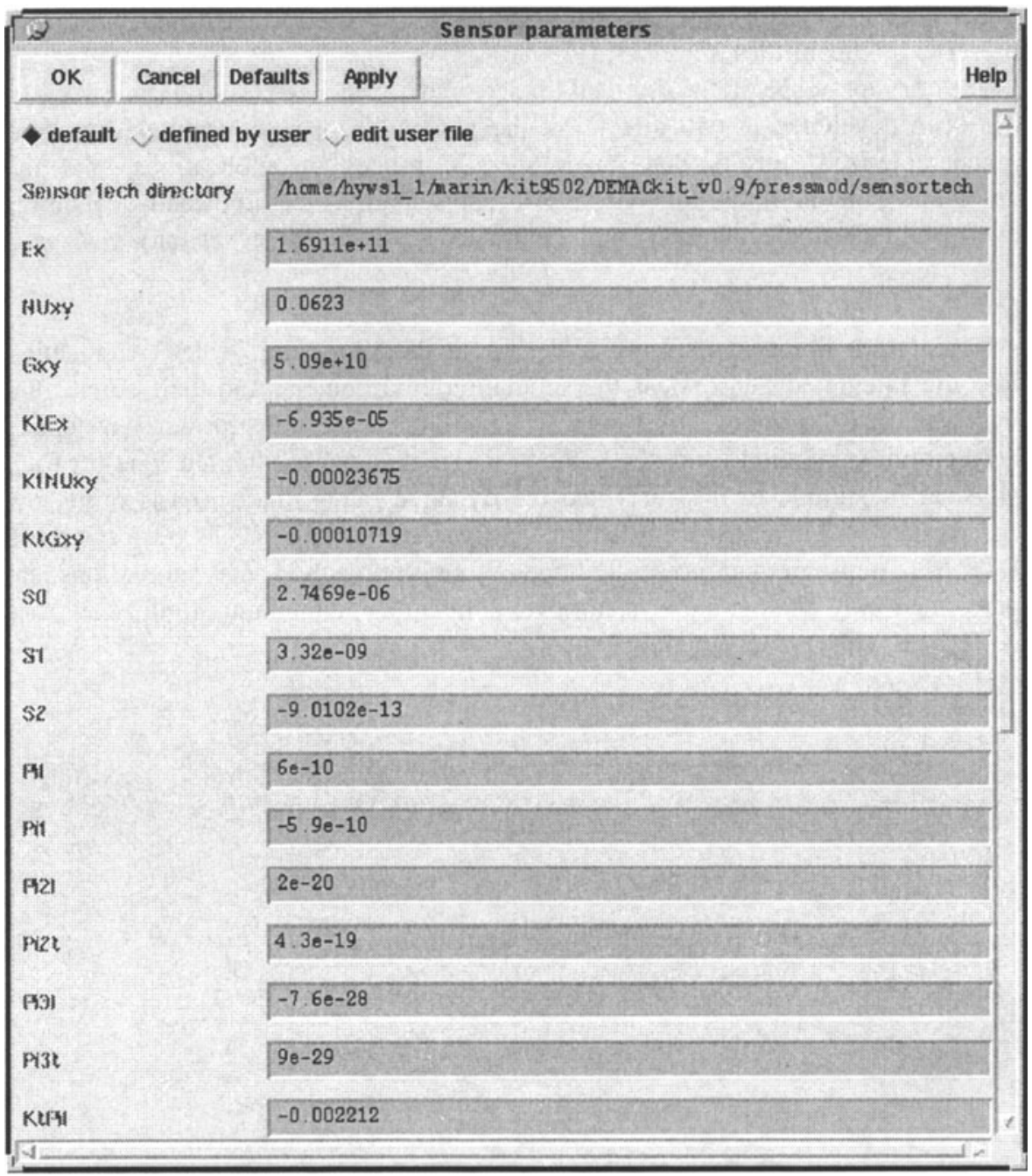

Figure 4. Fill form with pressure sensors characteristics inside Cadence DFWII.

\section{Sensor cell library}

At this point, it is important to note our main objective. We did not develop a complete new process, conversely we started from a standard process $(1 \mu \mathrm{m}$ from ATMEL-ES2) and introduced some additional steps. In this way, we enable the integration of different types of sensors together with all previously designed and characterized cells, that includes analog, digital pads, compiled structures, soft 
macros, etc. As a consequence, any new addition to the former design kit has to preserve its capabilities.

The concept of specific sensor cell library, under Cadence Design Framework II, has been developed in two directions: the generation of a set of predesigned an precharacterized sensors that cover specific ranges of applications, and the automatic generation tool for sensors. All representations required can be found for every cell (schematic, symbol, layout, extracted, abstract, spice, verilog).

\section{Simulation environment}

An important point in defining a design methodology for ASIS (Application Specific Integrated Sensors), is the simulation environment, and their connection with test and calibration. Those subjects are still open questions for microsystems.

At circuit simulation level, the number of signal sources is limited (one for each physical magnitude to measure) and the dynamic range of devices is quite low compared to MOS transistors (tenths of $\mathrm{kHz}$ ). The usual analysis performed are DC, AC, transient and noise. Following our approach, some simulations are performed only analog, and system level simulations, containing digital cells, are performed with mixed-signal tools. 


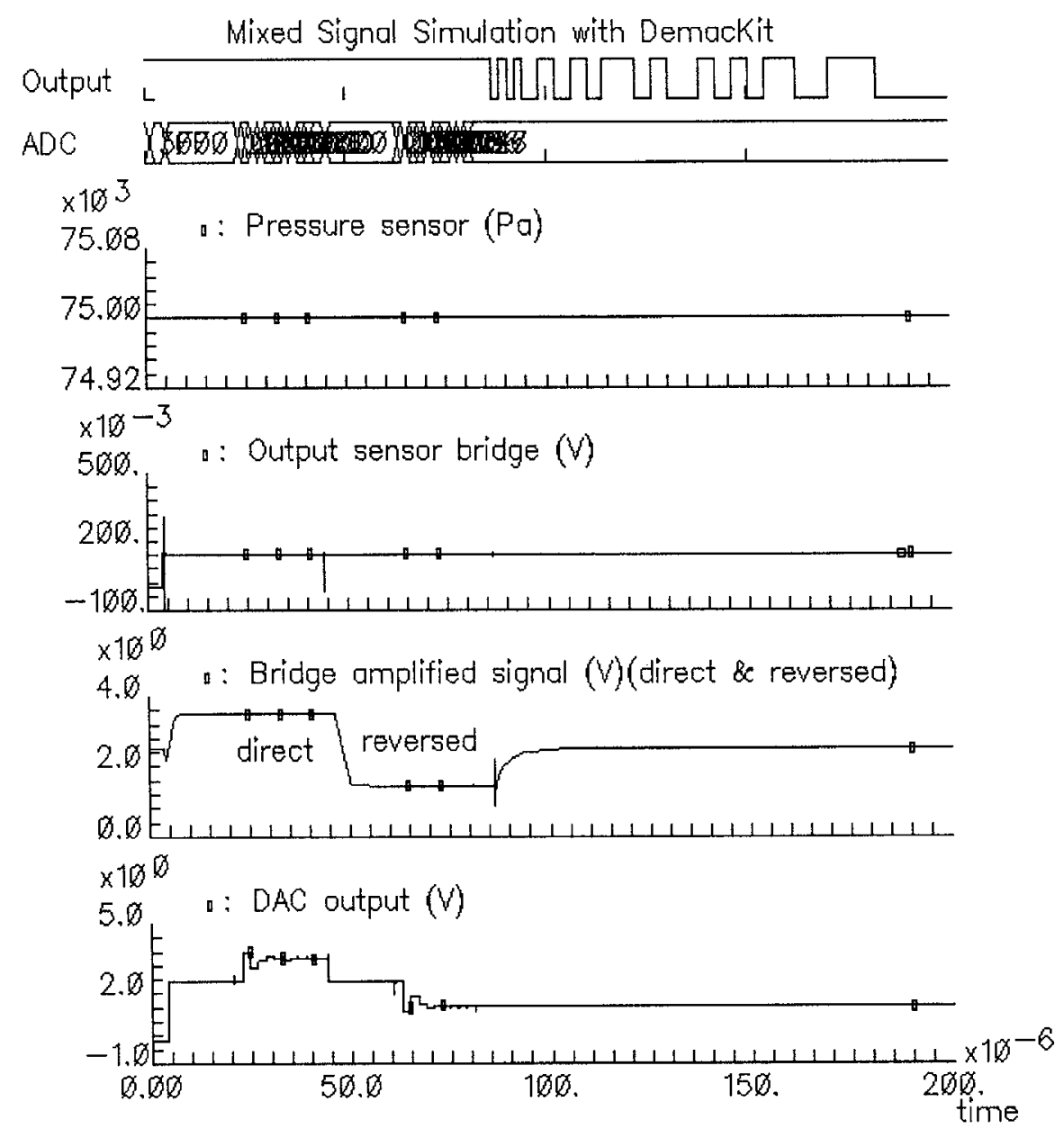

Figure 5. Mixed-signal simulation using pressure, analog and digital cells.

The choice not only depend on the availability of tools but also on the design kit offered by ATMEL-ES2. In this case, both analog and digital cells are represented by digital models, and basic simulation tool is based on Verilog. Then, we use mixed mode simulation for sensors and ad-hoc designed analog cells. In the case of specific design constrains, asking the foundry for spice models is required.

In terms of high-level simulation, we give basic Verilog models for sensors, but we mainly try to exploit mixed-signal environment, Analog Artist, in DFWII, by using electrical models for both sensors and analog cells (cdsspice, spectre or hspice) and structural for digital ones (Verilog), then some modifications to the current design kit were required. Figure 5 shows an example of such simulation. 


\section{CIRCUIT EXAMPLES}

For the ISFET case, a standard ISFET-meter structure has been built with ATMEL-ES2 analog cells. For this preliminary structure, sensor device and circuitry are placed one next to the other, as shown in Figure 6.

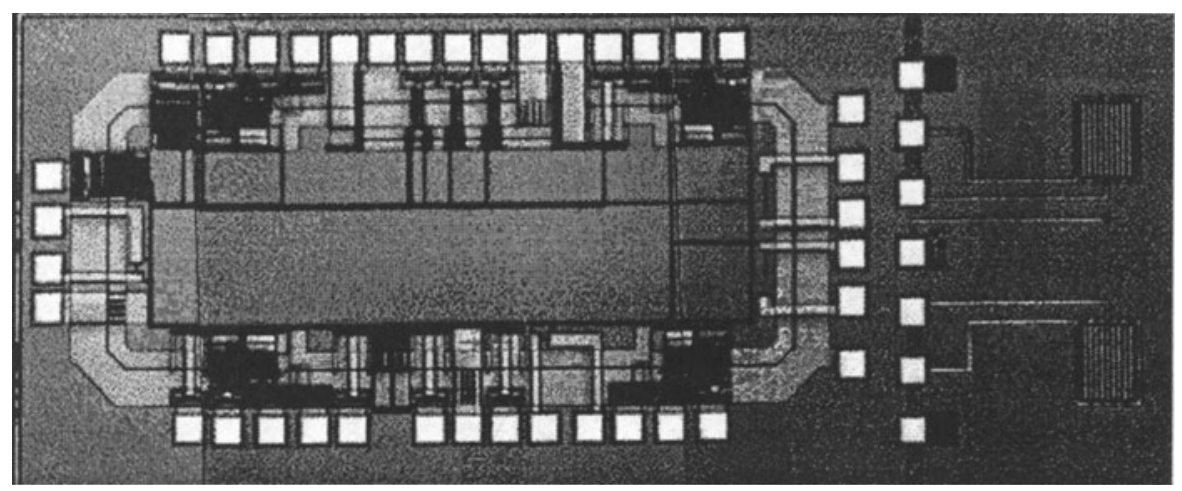

Figure 6. Photograph of the $\mathrm{pH}$-meter composed of sensors (right) and circuit (left)

Measurements of $\mathrm{pH}$ show acceptable results for this device, for range of operation (Figure 7) and sensitivity, $41 \mathrm{mV} / \mathrm{pH}$. The circuit is working according to specs.

\section{Atmel-ES2 Oxinitride ISFET}

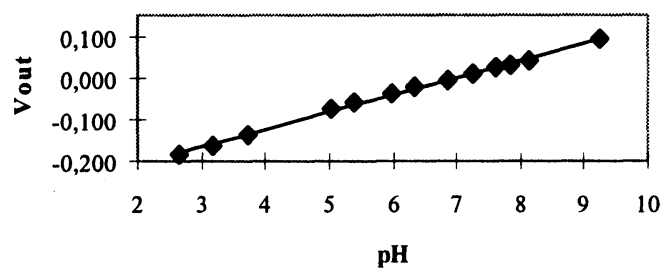

Figure 7. Measurement of range and sensitivity for fabricated ISFET devices.

They follow two different approaches. For the first one, $2 * 3 \mathrm{~mm}^{2}$ (Figure 8), analog and digital cells from ATMEL-ES2 have been used, and for the second one, $2.7 * 2.6 \mathrm{~mm}^{2}$, specially developed analog cells using current-mode techniques were used (designed by CNM Sevilla). Both microsystems perform pressure sensing, analog preprocessing, analog-to-digital conversion and digital coding in order to interface an standard microcontroller, 8051-like. Special plastic package has been developed by the customer, COPRECI, for this project. 


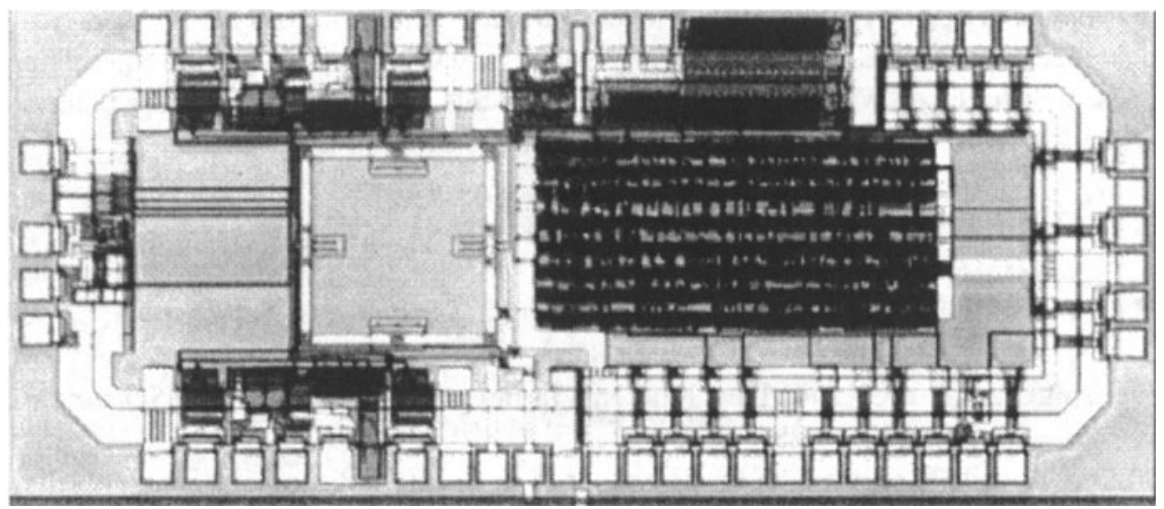

Figure 8. Photography of the integrated microsystem based on a pressure sensor.

The application environment is a prototype washing machine, composed of a water tank, the pressure sensor to obtain that measures the amount of water present in the tank, and the microcontroller. Preliminary measurements done by the industry, shows a resolution of 6 bits. Further advantages are noise reduction due to signal amplification closer to the sensor, and better control provided by digital coding for data transmission. As a consequence, industrialization is being considered.

\section{CONCLUSIONS}

Success in industrialization of integrated microsystems require to show the advantages of monolithic solutions: low extra cost compared with standard ASICs, reduction of area, better signal to noise ratio, few number of industries involved in the fabrication of the components of the whole system.

For a wide diffusion of this alternative, standardization of foundry processes and definition a clear design methodology is required. The objective of our work has been the development of specific cell libraries and design tools for the design of such microsystems. The manufacturing process is based on a standard CMOS technology, $1.0 \mu \mathrm{m}$ from ATMEL-ES2, and additional post-processing steps, done by CNM.

New concepts are required for the system design. Our approach tried to use as far as possible the same concepts used in mixed-signal circuit design. So, a standard commercial CAD tool, Cadence DFWII framework, is used.

A bottom-up strategy to develop process dependent data is proposed. Starting from the definition of the collection of design rules and basic set of cells, at fullcustom level. Following by the development and integration of a specific tool for device model compilation. And ending by the definition of a design methodology. 
By using this methodology several devices and microsystems have been designed and tested. Two of them, for $\mathrm{pH}$ and pressure measurements are presented together with some experimental results. Both show a correct behavior and allowed us to confirm the advantages of this new approach.

\section{REFERENCES}

Baltes H. (1993) CMOS as sensor technology. Sensors and Actuators A, 37-38 51-56.

Huijsing, J.H. et al. (1994) Developments in integrated smart sensors, Sensors and Actuators A, 43 276-288.

Marco S. (1993) Optimización de sensores de presión Piezoresisitivos de silicio para instrumentación biomédica y aplicaciones a alta temperatura. PhD Disertation. Universitat de Barcelona.

Merlos A. (1993) Desarrollo tecnológico para la Fabricación de ISFET con contactos posteriores $P h D$. Disertation. Universitat Autònoma de Barcelona.

Hebrard. L. (1995) Electrical models for piezoresistive pressure sensors Technical Report CNM.

Korvink J.G. and Baltes H. (1996) Microsystem CAD Proc. Micromachined Devices and Components II. SPIE. vol. 2882 pp. 170-181.

Karam, J.M. et al. (1996) Microsystem Design Framework based on Tool Adaptations and Library Developments Proc. Microlithography and Metrology in Micromachining II. SPIE. vol. 2880 pp. 236-245.

\section{BIOGRAPHY}

Authors belong to two different groups. The first one, headed by Dr. Carrabina, from $\mathrm{UAB}$, is specialized in cell libraries and design methodology for different types of technologies. Examples are available cell libraries for standard CMOS, SOI, DCVSL-logic, or tools for automatic cell library characterization, synthesis at logic and physical level, and circuit formal verification at switch level.

The second one, headed by Dr. Bausells, from CNM-IMB, and is devoted to sensor process development, device modeling and industrial implementation of microsystems. 